\title{
Unblended disjoint tree merging using GTM improves species tree estimation
}

\author{
Vladimir Smirnov and Tandy Warnow* \\ From 17th RECOMB Satellite Conference on Comparative Genomics \\ Montpellier, France. 1-4 October 2019
}

\begin{abstract}
Background: Phylogeny estimation is an important part of much biological research, but large-scale tree estimation is infeasible using standard methods due to computational issues. Recently, an approach to large-scale phylogeny has been proposed that divides a set of species into disjoint subsets, computes trees on the subsets, and then merges the trees together using a computed matrix of pairwise distances between the species. The novel component of these approaches is the last step: Disjoint Tree Merger (DTM) methods.

Results: We present GTM (Guide Tree Merger), a polynomial time DTM method that adds edges to connect the subset trees, so as to provably minimize the topological distance to a computed guide tree. Thus, GTM performs unblended mergers, unlike the previous DTM methods. Yet, despite the potential limitation, our study shows that GTM has excellent accuracy, generally matching or improving on two previous DTMs, and is much faster than both.

Conclusions: The proposed GTM approach to the DTM problem is a useful new tool for large-scale phylogenomic analysis, and shows the surprising potential for unblended DTM methods.
\end{abstract}

Keywords: Large-scale phylogeny estimation, Species tree estimation, Divide-and-conquer pipelines

\section{Background}

The estimation of evolutionary trees (i.e., phylogenies, whether of genes or of species) is a fundamental step in much biological research, including understanding how species adapt to their environments, how gene function evolves, and how humans migrated across the globe. Yet, phylogeny estimation is computationally intensive, as nearly all the best approaches are based on NP-hard optimization problems.

Divide-and-conquer approaches to tree estimation have the potential to provide improved scalability to heuristics for NP-hard optimization problems, but they depend on supertree methods, which have not been established to be highly scalable [1]. A new divide-and-conquer approach

*Correspondence: warnow@illinois.edu Department of Computer Science, University of Illinois at Urbana-Champaign, 201 N Goodwin Ave, 61801 Urbana, IL, US has been recently proposed to tackle this limitation: the set of species is divided into smaller, disjoint subsets, trees are computed on each subset (using the best available methods), and then the trees are combined together into a tree on the complete taxon set. In this approach, the subset trees are considered hard constraints and so must be induced in the output tree [2-6]; this property of the output tree is expressed by saying it is a "compatibility supertree" of the input constraint trees. Finally, merging disjoint trees requires some auxiliary information (such as a matrix of pairwise distances between the species), and the problem of merging disjoint trees using auxiliary information is called the "Disjoint Tree Merger" (DTM) problem.

So far, three DTM methods have been developed: NJMerge [2, 3], TreeMerge [4], and constrained-INC [5, 6], each of which uses a computed matrix of pairwise distances to merge the disjoint trees and does so in

(c) The Author(s). 2020 Open Access This article is licensed under a Creative Commons Attribution 4.0 International License, which permits use, sharing, adaptation, distribution and reproduction in any medium or format, as long as you give appropriate credit to the original author(s) and the source, provide a link to the Creative Commons licence, and indicate if changes were made. The images or other third party material in this article are included in the article's Creative Commons licence, unless indicated otherwise in a credit line to the material. If material is not included in the article's Creative Commons licence and your intended use is not permitted by statutory regulation or exceeds the permitted use, you will need to obtain permission directly from the copyright holder. To view a copy of this licence, visit http://creativecommons.org/licenses/by/4.0/. The Creative Commons Public Domain Dedication waiver (http://creativecommons.org/publicdomain/zero/1.0/) applies to the data made available in this article, unless otherwise stated in a credit line to the data. 
polynomial time. Constrained-INC was tested only for gene tree estimation, where it had disappointing results. TreeMerge and NJMerge were tested in the context of species tree estimation on multi-locus datasets, where they provided good advantages: they matched the accuracy of leading species tree estimation methods (ASTRAL [7-9] and RAxML [10], which performs a concatenation maximum likelihood analysis), while providing improvements in speed. NJMerge can fail to return a tree under some conditions (though never when merging two trees), due to its algorithmic design $[2,3]$; TreeMerge is a technique that uses NJMerge on pairs of trees (where it is guaranteed to never fail), and then combines overlapping trees using an innovative algorithm that exploits the ability to estimate branch lengths. Thus, TreeMerge was developed specifically to replace NJMerge because of the capacity of NJMerge to fail and in order to improve the speed. Finally, all three DTM methods have been proven to enable statistically consistent tree estimation when used within appropriate divide-and-conquer strategies.

We propose a new DTM approach, where the input is a set of disjoint trees and also a tree on the full set of species (called a "guide tree"), and the objective is to produce a tree that agrees with all constraint trees and minimizes the total topological distance to the guide tree. We prove this optimization problem is NP-hard, but show that if we do not allow blending (so that the output tree is formed by just adding edges between the constraint trees), then the problem is solvable in polynomial time.

We present the Guide Tree Merger (GTM) algorithm to solve this unblended DTM-GT problem, and prove that it uses polynomial time. We evaluate GTM for species tree estimation from multi-locus datasets, where gene trees can differ from the species tree due to incomplete lineage sorting [11]. We prove that the pipeline using GTM (Fig. 1) maintains statistical consistency, and demonstrate (on a collection of multi-locus datasets with 1000 species) that GTM has very good performance. Specifically, we show that GTM matches or improves on the accuracy of NJMerge and TreeMerge on these datasets, while completing in a fraction of their runtimes. See
Additional file 1 for details for the experimental performance study, Additional file 2 for proofs, and Additional files 3 and 4 for additional figures and tables, respectively.

\section{The DTM-GT optimization problem}

All phylogenetic trees are assumed to be unrooted and leaf-labelled by taxa in a set $S$. We continue with some basic terminology.

Given a tree $T$ on leafset $S$ and set $S^{\prime} \subseteq S$, the homeomorphic subtree of $T$ defined by $S^{\prime}$ (in which degree two nodes are suppressed) is denoted by $\left.T\right|_{S^{\prime}}$.

Definition 1 Let $\mathcal{A}=\left\{T_{1}, T_{2}, \ldots, T_{k}\right\}$ be a set of trees on subsets of $S$. $T$ is said to be a compatibility supertree for $\mathcal{A}$ if (1) $L(T)=\cup_{i} L\left(T_{i}\right)$ (where $L(t)$ denotes the leaf set of $t$ ) and (2) $\left.T\right|_{L(t)}=t$ for all $t \in \mathcal{A}$.

Each edge $e$ in a phylogenetic tree $t$ defines a bipartition $\pi_{e}$ on the leafset of $t$, and hence each tree $t$ is defined by the set of its bipartitions, which we denote by $C(t)=$ $\left\{\pi_{e} \mid e \in E(T)\right\}$. The "FN" (false negative) distance of tree $A$ to tree $B$ is $|C(B) \backslash C(A)|$, or the number of bipartitions in $C(B)$ that are missing from $C(A)$, and is denoted by $F N(A, B)$.

We continue with a discussion of DTM methods. Each DTM method takes as input a set $\mathcal{T}$ of leaf-disjoint trees and some auxiliary information and returns a compatibility supertree for $\mathcal{T}$. Note that since the trees in $\mathcal{T}$ are on disjoint leaf sets, a compatibility supertree is guaranteed to exist (e.g., the tree formed by including a center node $v$ and making $v$ adjacent to some internal vertex in each of the trees in $\mathcal{T}$ is a compatibility supertree). The key to making DTM methods have good accuracy is the use of auxiliary information to merge the trees together well.

The current DTM methods perform this merger by computing a matrix of pairwise distances between species under a statistical model of evolution, and then carefully use the resultant matrix while merging the subset trees. For example, NJMerge accomplishes this by modifying the popular Neighbor Joining method [12], which agglomeratively builds the tree, so that it never violates any subset

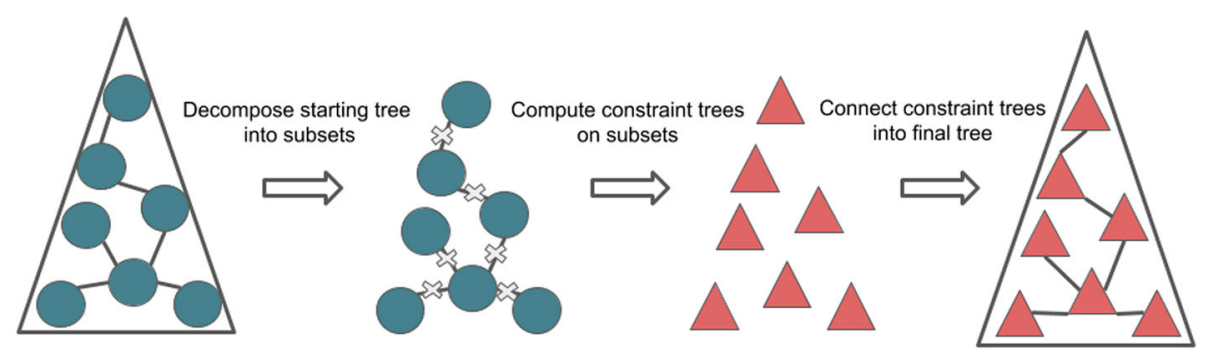

Fig. 1 A basic DTM pipeline with GTM. A starting tree is computed and then decomposed by deleting some set of edges. Constraint trees are computed for these subsets and attached together with new edges using GTM 
tree in $\mathcal{T}$. Furthermore, the current DTM methods allow blending, by which we mean that the subset trees in $\mathcal{T}$ may not be separated from each other within the compatibility supertree.

To understand why blending can be necessary, suppose $D$ is an additive matrix (i.e., there is an edge-weighted tree $T$ so that $D[x, y]$ is the distance between $x$ and $y$ in $T$ ) that defines a caterpillar tree on 8 leaves, i.e., $T=(1,(2,(3,(4,(5,(6,(7,8)))))))$. Now suppose $t_{1}=$ $(1,(5,(6,7)))$ and $t_{2}=(2,(3,(4,8)))$. Now consider when the input to the DTM method is the pair $\mathcal{T}=\left\{t_{1}, t_{2}\right\}$ and $D$. The desired output should be $T$, which is a compatibility supertree for $\mathcal{T}$ that realizes the input matrix $D$. Yet, $T$ can only be formed if $t_{1}$ and $t_{2}$ are blended together; thus, unblended merges (formed by adding an edge connecting the input trees) are not as powerful as blended mergers.

In this paper, we introduce GTM (Guide Tree Merger), a new DTM method that does not allow blending. Despite this limitation, we will show GTM matches or improves on the previous DTM methods NJMerge and TreeMerge under most tested conditions, and is much faster than both. Before we show our method, we introduce an optimization problem for DTM construction.

\section{Definition 2 DTM-GT-FN:}

- Input: $A$ set $\mathcal{T}=\left\{T_{1}, T_{2}, \ldots, T_{k}\right\}$ of leaf-disjoint unrooted binary trees and a guide tree $T^{*}$ on the full set of species

- Output: A compatibility supertree $T$ for $\mathcal{T}$ that minimizes $\left|C\left(T^{*}\right) \backslash C(T)\right|$ (i.e., $T$ minimizes the $F N$ distance to $\left.T^{*}\right)$.

Here, GT refers to "guide tree" and "FN" refers to the optimization criterion, which is that we wish to minimize the FN (false negative) distance between the guide tree and the compatibility supertree we construct. We allow the trees in the input set $\mathcal{T}$ to be non-binary, and for this reason we use the FN distance rather than the RF distance. However, when the guide tree and all the trees in $\mathcal{T}$ are binary, then the optimal compatibility supertree is also binary, and the RF distance and FN distance to the guide tree are identical.

\section{Theorem 1 DTM-GT-FN is NP-hard}

See Additional file 2 for the proof. Now consider the following variant of DTM-GT-FN, where we do not permit blending:

\section{Definition 3 Unblended DTM-GT-FN:}

- Input: $A$ set $\mathcal{T}=\left\{T_{1}, T_{2}, \ldots, T_{k}\right\}$ of leaf-disjoint unrooted binary trees and a guide tree $T^{*}$ on the full set of species
- Output: A compatibility supertree $T$ for $\mathcal{T}$ formed by connecting the trees in $\mathcal{T}$ by edges and that minimizes $\left|C\left(T^{*}\right) \backslash C(T)\right|$ (i.e., the $F N$ distance to $\left.T^{*}\right)$ among all such compatibility supertrees.

Interestingly, Unblended-DTM-GT-FN can be solved in polynomial time, as we show in the next section.

\section{The GTM method}

We begin with some terminology. First, we say that a bipartition $\pi=A \mid B$ "violates a constraint tree" $t$ if $t$ does not contain any edge defining the bipartition $\left.\pi\right|_{L(t)}=$ $A \cap L(t) \mid B \cap L(t)$, where $\left.\pi\right|_{L(t)}$ denotes $\pi$ restricted to $L(t)$. Next, we refer to the leaf sets of each constraint tree as "constraint sets", and we partition the edges of the guide tree $T^{*}$ into three sets, based on how many constraint sets $(0,1$, or at least 2$)$ have leaves on both sides of the edge.

- The edges that have no constraint sets on both sides are called "bridge edges", as they separate constraint sets.

- The edges that have exactly one constraint set on the two sides are said to "lie within that constraint set."

- The edges that have two or more constraint sets on the two sides are said to "violate convexity" (where convexity would assert that the nodes of the guide tree can be labelled by the constraint sets, so that no node has more than one label and every two leaves with the same label are connected by a path with all nodes given the same label).

GTM solves the Unblended DTM-GT-FN problem recursively, in polynomial time, as described below. Specifically, GTM uses the guide tree to determine which pairs of constraint trees should be connected directly by edges, and then uses bipartitions in the guide tree to determine how to connect the constraint trees together; this depends on finding appropriate edges in each constraint tree to subdivide (by adding internal nodes) and then which pairs of constraint trees should be connected by new edges (introduced between the newly created internal nodes in the subdivided edges). The details of how these edges are identified and how the specific pairs of constraint trees are merged are non-trivial, and are described below:

The GTM algorithm has the following structure:

- Collapse all edges in $T^{*}$ that violate any $T_{i}$.

- Collapse all edges in $T^{*}$ that violate convexity.

- Return Rejoin $\left(T^{*}\right)$.

Note that $\operatorname{Rejoin}(\cdot)$ is run only after all edges that violate convexity or a constraint tree have been collapsed. Hence, every remaining edge is either a bridge edge (i.e., separates constraint sets) or is on a path between two leaves for exactly one constraint subset (i.e., "lies within" their 


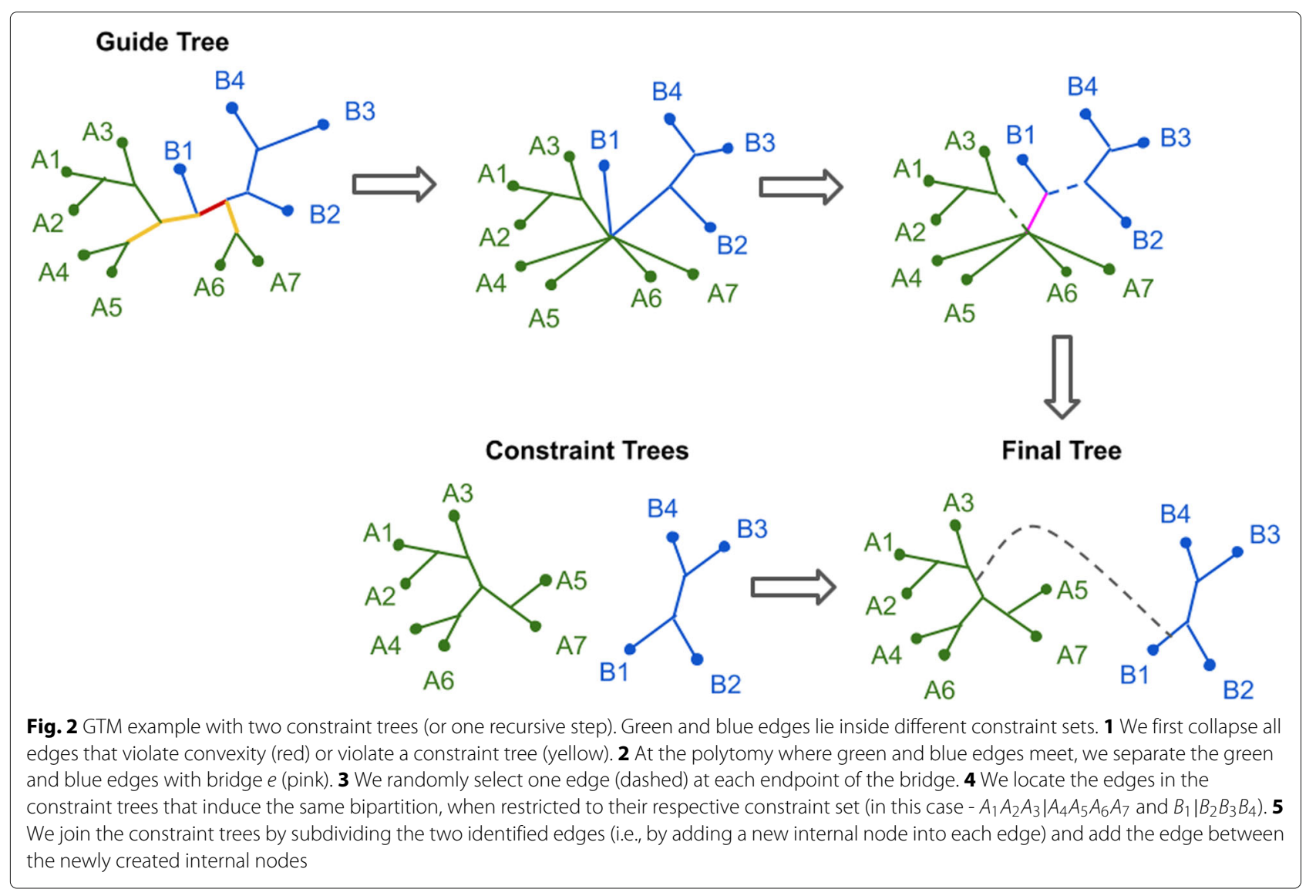

constraint sets). Furthermore, every edge $e$ that lies within constraint set $L\left(T_{i}\right)$ satisfies $b(e):=\left.\pi(e)\right|_{L\left(T_{i}\right)} \in C\left(T_{i}\right)$ (as otherwise it would have violated the constraint tree and would have been collapsed). Given this context, we now describe the recursive function $\operatorname{Rejoin}(T)$ (Fig. 2):

$\operatorname{Rejoin}(T)$ :

- If $L(T)=L\left(T_{i}\right)$ for some $i$, Return $T_{i}$.

- Pick an edge $e$ in $T$ as follows. Let $v \in T$ be any "border node", i.e., a node that is either (1) an endpoint of a bridge edge, or (2) a common endpoint of two edges lying in different constraint sets $L\left(T_{i}\right)$ and $L\left(T_{j}\right)$. Given $v$, we define edge e as follows: in case (1), e is the bridge edge that $v$ is incident with, and in case (2), we refine at node $v$ by adding a vertex $v^{\prime}$ and new edge $\left(v, v^{\prime}\right)$ to separate all the edges incident with $v$ that lie in $L\left(T_{i}\right)$ from all the other edges, and set $e=\left(v, v^{\prime}\right)$.

- Delete e from $T$ to form two trees, $S_{1}$ and $S_{2}$. /*Comment: The species in each constraint subset are in exactly one subtree, $S_{1}$ or $S_{2}$.

- Let edges $e_{1} \in E\left(S_{1}\right)$ and $e_{2} \in E\left(S_{2}\right)$ be any two edges that are incident with $e$.

- Let $S_{1}^{\prime}=\operatorname{Rejoin}\left(S_{1}\right)$ and $S_{2}^{\prime}=\operatorname{Rejoin}\left(S_{2}\right)$. /*Comment: Note that $S_{1}^{\prime}$ and $S_{2}^{\prime}$ are both unblended compatibility supertrees of some of the constraint trees, and each constraint tree appears within one of these two trees. ${ }^{*} /$

- Find "attachment edges" $e_{i}^{\prime}$ in trees $S_{i}^{\prime}(i=1,2)$, such that $e_{i}$ and $e_{i}^{\prime}(i=1,2)$ define the same bipartitions in their respective trees.

- Join $S_{1}^{\prime}$ and $S_{2}^{\prime}$ by "adding an edge between $e_{1}^{\prime}$ and $e_{2}^{\prime}$ " (i.e., by subdividing $e_{1}^{\prime}$ and $e_{2}^{\prime}$, thus creating new nodes $v_{1}$ and $v_{2}$ and adding edge $\left.\left(v_{1}, v_{2}\right)\right)$ and return the resulting tree.

In other words, for each pair of constraint trees to be joined, we add an edge to connect them by choosing one edge from each tree to bridge together. This "attachment edge" is chosen by finding an edge with the same bipartition, relative to the subtree, as any of the edges at the attachment point of that subtree in $T^{*}$.

Theorem 2 Given a guide tree $T^{*}$ and set $\mathcal{T}=$ $\left\{T_{1}, T_{2}, \ldots, T_{k}\right\}$ of constraint trees over their respective leaf sets $L\left(T_{i}\right)$ of $T_{i}^{*}$, GTM optimally solves Unblended DTMGT-FN on $\left(\mathcal{T}, T^{*}\right)$, and has a worst case running time complexity of $O\left(N^{2}\right)$, where $N=\left|L\left(T^{*}\right)\right|$ is the total number of species. 
We sketch the proof here, and direct the reader to Additional file 2 for full details. We note that another way of describing GTM is that it collapses all edges in the guide tree that violate convexity or violate a constraint tree, it adds edges to separate the constraint sets, and then it refines the resultant tree to induce each of the constraint trees. As a result, it is easy to see that it maximizes the number of shared bipartitions, and so equivalently it minimizes the number of missing branches in $T^{*}$. Hence, it optimally solves Unblended DTM-GT-FN. For the running time, we add that to achieve the $O\left(N^{2}\right)$ time complexity, the algorithm uses hashing.

\section{Divide-and-conquer pipelines for DTMs}

Here we describe a generalization of the divide-andconquer pipelines that were previously used to evaluate DTMs in $[2-4,6]$ in the context of multi-locus species tree estimation and gene tree estimation.

- Construct a starting tree $T_{0}$ using a fast but statistically consistent method, $X$

- Divide the species set into disjoint sets by decomposing the tree $T_{0}$ (by deleting edges) until each subset has at most $k$ species

- Construct trees on each subset using the preferred method, $Y$

- Combine the subset trees together using a DTM method, $Z$

We will refer to a given pipeline for multi-locus species tree estimation as the triple " $X-Y-Z$ "; for example "NJstRAxML-TreeMerge" indicates that NJst [13] is used to compute the starting tree, RAxML is used to construct subset trees, and TreeMerge is used to combine the subset trees into one tree. In our experiments, we repeatedly delete edges from the starting tree until all the subsets have at most 120 species.

In the experiments we report in this paper, we use the centroid edge decomposition strategy to divide our species sets into disjoint subsets; this is the same decomposition strategy used in the NJMerge $[2,3]$ and TreeMerge [4] studies. The centroid edge decomposition technique originated in SATé-II [14] and has also been used in PASTA $[15,16]$ and DACTAL [17]. The basic objective of this strategy is to produce a decomposition where every subset is "local" within the input tree, and does not exceed a specified maximum subset size. The centroid edge decomposition is based on finding and deleting centroid edges, which are edges whose removal splits the leaf set into two sets where the difference in size is minimized among all the edges in the tree; because there can be more than one edge with this property, the centroid edge may not be unique. In the centroid edge decomposition strategy, a tree $T$ is given as well as a bound $B$ on the size of the subsets to be computed. If $T$ has at most $B$ leaves, then the set of leaves of $T$ is returned (i.e., there is no decomposition performed). Otherwise, a centroid edge is found and removed, and then the algorithm recurses on the two subtrees. At the end of this recursive strategy, the set of leaves of $T$ (i.e., taxa) is decomposed into disjoint sets, each of which has size at most $B$.

A desirable property of a phylogeny estimation method or pipeline is that it is statistically consistent, which means that it provably converges to the true tree as the amount of data increases. Pipelines using NJMerge and TreeMerge were proven statistically consistent under the MSC+GTR model $[3,4]$ where gene trees evolve within the species tree under the multi-species coalescent (MSC) model [18] and then sequences evolve down each gene under the Generalized Time Reversible (GTR) model [19]) and a similar pipeline using constrained-INC was proven to be statistically consistent under the GTR model [5]. The following theorem shows that appropriate divideand-conquer pipelines using GTM are also statistically consistent; see Additional file 2 for the proof.

Theorem 3 Let $\Phi$ be a model of evolution. If the method $X$ used to construct the starting tree and the method $Y$ used to construct the subset trees are both statistically consistent under $\Phi$, then the DTM pipeline X-Y-GTM is also statistically consistent under $\Phi$.

Hence, NJst-ASTRAL-GTM is statistically consistent under the MSC+GTR model, but RAxML, FastTree2 [20], and any divide-and-conquer strategies based on these methods will not be (because maximum likelihood is not statistically consistent under the MSC+GTR model [21, 22]). Note that statistical consistency does not depend on using the centroid edge decomposition, and instead is guaranteed for any decomposition strategy that operates by removing a set of edges from the starting tree and computing subset trees on the leaf sets of the resulting components.

The terms "starting tree" and "guide tree" can have somewhat different meanings in divide-and-conquer pipelines. The starting tree is used to produce subsets, while the guide tree is part of the input to the GTM algorithm. Within our " $X-Y$-GTM" pipelines, the starting tree $(X)$ and the guide tree are always the same, permitting the terms to be used interchangeably in this context. However, other pipelines can be considered where the decomposition into subsets is based on a starting tree and then the subset trees are merged using a guide tree that is different from the starting tree.

\section{Experimental study}

We give an overview of the experimental study here; see Additional file 1 for additional details and software commands used in this study. 


\section{Overview}

We compare GTM to NJMerge and TreeMerge with respect to species tree error using previously published multi-locus datasets with 1000 species and varying numbers of genes where gene trees can differ from the true species tree due to incomplete lineage sorting. We used multi-locus datasets and estimated gene trees (computed using FastTree2) from [3].

We vary the starting tree and method to compute constraint trees, so as to explore the impact of these choices on accuracy of the final tree. We compare each estimated species tree to the true species tree, and report the normalized Robinson-Foulds (RF) error rates [23], where the RF error of tree $T$ is the total number of bipartitions that appear in $T$ or the true tree but not both (RF distance), divided by $2 N-6$, where $N$ is the number of leaves (note that $2 N-6$ is the total number of non-trivial bipartitions if both trees are binary). Thus, the RF error ranges from 0 (perfect match) to 1 (nothing in common). We also report the running time of the pipelines, and compare them to the running time for standard species tree estimation methods on the same datasets.

We explore GTM within the same divide-and-conquer pipeline strategy used in [3, 4], as described above, using the centroid edge decomposition and decomposing until each subset has at most 120 species.

\section{External methods}

We compare the trees computed using the pipelines to unpartitioned maximum likelihood using RAxML and FastTree2; we also computed trees on the multi-locus datasets using summary methods (i.e., methods that estimate the species tree by combining the gene trees) ASTRAL, NJst [13], and ASTRID [24]. NJst, ASTRID, and ASTRAL are statistically consistent under the MSC+GTR model, but FastTree 2 and RAxML are not.

The divide-and-conquer pipelines require external methods to compute starting trees and constraint trees: we used FastTree2, NJst, and ASTRAL for the starting trees, and ASTRAL and RAxML for the constraint trees. We include ASTRAL and RAxML because these are the current leading methods for species tree estimation on large datasets. We include NJst and ASTRID because they, like ASTRAL, are species tree estimation methods that are polynomial time, statistically consistent under the MSC model, and can run on 1000-species datasets. Finally, we include FastTree2 (henceforth called FastTree) because it is a very fast maximum likelihood heuristic.

\section{Datasets}

Our study makes use of the 1000-taxon multi-locus simulated datasets from the NJMerge study [3], which are available at [25]. Here we briefly describe the experimental protocol used in [3], and how we used the datasets to test GTM pipelines.

Each replicate dataset in [3] has a true species tree and 1000 true gene trees. The gene trees were generated by evolving them down the species tree under the MSC model, which has the consequence that the true gene trees can differ from the true species tree due to incomplete lineage sorting (ILS). The sequence alignments in [3] were produced by evolving sequences down the true gene trees under the GTR model and gene trees were estimated on these alignments using FastTree [26]. The study in [3] includes model species trees with different levels of ILS and two types of genes (exons and introns) that have two different rates of evolution. Among the datasets available from [3], we selected 1000-taxon datasets from model conditions with two levels of ILS (low and very high) and both types of genes.

In order to stress test GTM, we explored conditions where estimating a reasonably accurate starting tree might be difficult. Since the number of genes impacts the accuracy of the starting tree (especially when ILS is high), we achieved this by including analyses with only 10 and 25 genes, which we selected by picking the first such genes from the data repository for the study.

We explored a range of fast methods for computing the starting tree, including FastTree (which can only be used when the number of genes is not too large), ASTRAL, and NJst [13]. The result was a set of starting trees that varied in accuracy, from very accurate to very inaccurate. In general, all starting trees on low ILS conditions were reasonably accurate, even with only 10 genes. However, with high ILS conditions and ten genes, all starting trees had low accuracy: the best starting trees had error rates in the $50-60 \%$ RF error range and the worst starting trees (computed using ASTRAL) had error rates between 64-66\% (see Additional files 3 and 4). Thus, all the starting trees for the 10-gene high ILS conditions are poor, and they provide the stress test we need to understand how GTM operates when given poor starting trees.

Table 1 Dataset properties

\begin{tabular}{ll}
\hline $\begin{array}{l}\text { Number of species } \\
\text { Number of sites per gene }\end{array}$ & 1000 \\
\hline Number of genes & $300-1500$ \\
ILS levels & $10,25,1000$ \\
& Very High ILS (68-69\% AD) \\
Lene types & Low ILS (8-10\% AD) \\
& Exons (38-64\% GTEE) \\
& Introns (26-51\% GTEE) \\
\hline
\end{tabular}

$A D$ values of ILS levels are the average Robinson-Foulds distances between the true species tree and the true gene trees. Gene Tree Estimation Error (GTEE) is the average RF error of the estimated gene trees 
Overall, we considered 12 model conditions (three numbers of genes, two types of genes, and two levels of ILS), and each model condition has 20 replicates (see Table 1).

\section{Experiments and computational resources}

We performed three experiments. Experiment 1 determined the best way to compute starting trees and subset trees for each DTM method (NJMerge, TreeMerge, and GTM) within the divide-and-conquer pipeline, for each model condition (i.e., ILS level and number/type of genes). An important part of this experiment is that it allowed us to evaluate the impact of the starting tree on GTM, which is a matter of some interest to us. Experiment 2 compared the three DTM methods to each other with respect to accuracy and running time. Experiment 3 evaluates the impact of divide-and-conquer pipelines using GTM on RAxML and ASTRAL, the two leading species tree estimation methods, with respect to accuracy and running time.

All the analyses we performed were executed on the Campus Cluster at UIUC, which limits analyses to 4 hours; hence, any analysis that failed to complete within that time was discarded. However, for the 1000-gene analyses involving NJMerge and RAxML (which could not complete within 4 hours on the Campus Cluster), we report results given in [3] that used the Blue Waters supercomputer and allowed to run for 48 hours.

\section{Results}

Missing replicates in the figures typically reflect NJMerge (and in some cases ASTRAL and RAxML) failures to complete within the allowed running time (see Additional file 4).

\section{Experiment 1: designing the DTM pipelines}

We consider the accuracy of pipelines for each DTM method, selecting either RAxML or ASTRAL for constraint trees and NJst, ASTRAL, or FastTree for the starting tree (where they can be run within the stated time limits).

Results for TreeMerge and GTM on the high ILS datasets with 10 introns are shown in Fig. 3; NJMerge is not shown for these data, as it failed for all these replicates (as well as for the analyses with 25 high ILS introns). This experiment shows that the most accurate results are obtained using NJst-ASTRAL-TreeMerge and NJst-ASTRAL-GTM (which are tied), and all other combinations have distinctly higher error. Interestingly, the next best analyses are obtained using FastTree-ASTRALTreeMerge and FastTree-ASTRAL-GTM (which are tied), but ASTRAL-ASTRAL-TreeMerge and ASTRAL-ASTRALTM. have much higher error rates. Finally, the least accurate results are obtained using ASTRAL-RAxMLTreeMerge and ASTRAL-RAxML-GTM. Figure 3 also shows that the starting trees obtained using ASTRAL and constraint trees obtained using RAxML have very high error on these data, which explains these negative results. Results for other high ILS conditions show the same trends; see Additional file 3. Overall, therefore, the most accurate analyses on high ILS datasets are obtained using NJst-ASTRAL-TreeMerge and NJst-ASTRAL-GTM.

Results for low ILS datasets with 10 introns show different trends (Fig. 4). Here we see that the most accurate results are obtained using FastTree-RAxMLGTM, with FastTree-RAxML-NJMerge slightly less accurate (followed by FastTree-RAxML-TreeMerge). The next best results are obtained using other combinations with RAxML to compute the constraint trees, which is explained by noting that the RAxML constraint trees are highly accurate. We also see that the FastTree starting tree is highly accurate, which helps explain the good performance of FastTree-RAxML-GTM. Results for other low ILS conditions are shown in see Additional file 3, and show the same trends. Overall, for low ILS datasets, the best accuracy is obtained using FastTree-RAxMLGTM. However, FastTree cannot run to completion on 1000-gene datasets, which limits its utility as a general starting tree to only those datasets with not too many genes. Therefore, for 1000-gene datasets, we recommend NJst-RAxML-GTM.

In order for the pipeline to be provably statistically consistent under the MSC+GTR model, both the starting tree and the constraint trees must be computed using methods that are statistically consistent under the MSC+GTR model, which means (for our study) either ASTRAL or NJst for both steps. NJst is much faster than ASTRAL, and can complete on all the datasets we explored within the limited allowed time (which is not true for ASTRAL). Therefore, when statistical consistency is required, NJstASTRAL is the right pipeline for all DTM methods.

\section{Experiment 2: comparing DTM methods}

We compare the three DTM methods on 1000-species datasets. We show results for the NJst-RAxML and NJstASTRAL pipelines, but results for other pipelines show similar trends.

Under the low ILS conditions, GTM and NJMerge have very similar RF error rates, with TreeMerge slightly higher in error (Fig. 5). For the high ILS conditions, the comparison is made between GTM and TreeMerge, as NJMerge failed to complete on many datasets (see Additional file 4). TreeMerge and GTM have very close accuracy under most high ILS conditions, and neither dominates the other method (Additional file 3).

Runtime performance is compared in Table 2 for high ILS and Table 3 for low ILS. Under conditions where NJMerge and TreeMerge both run, NJMerge is slower, and each takes many minutes (up to 30 minutes for 

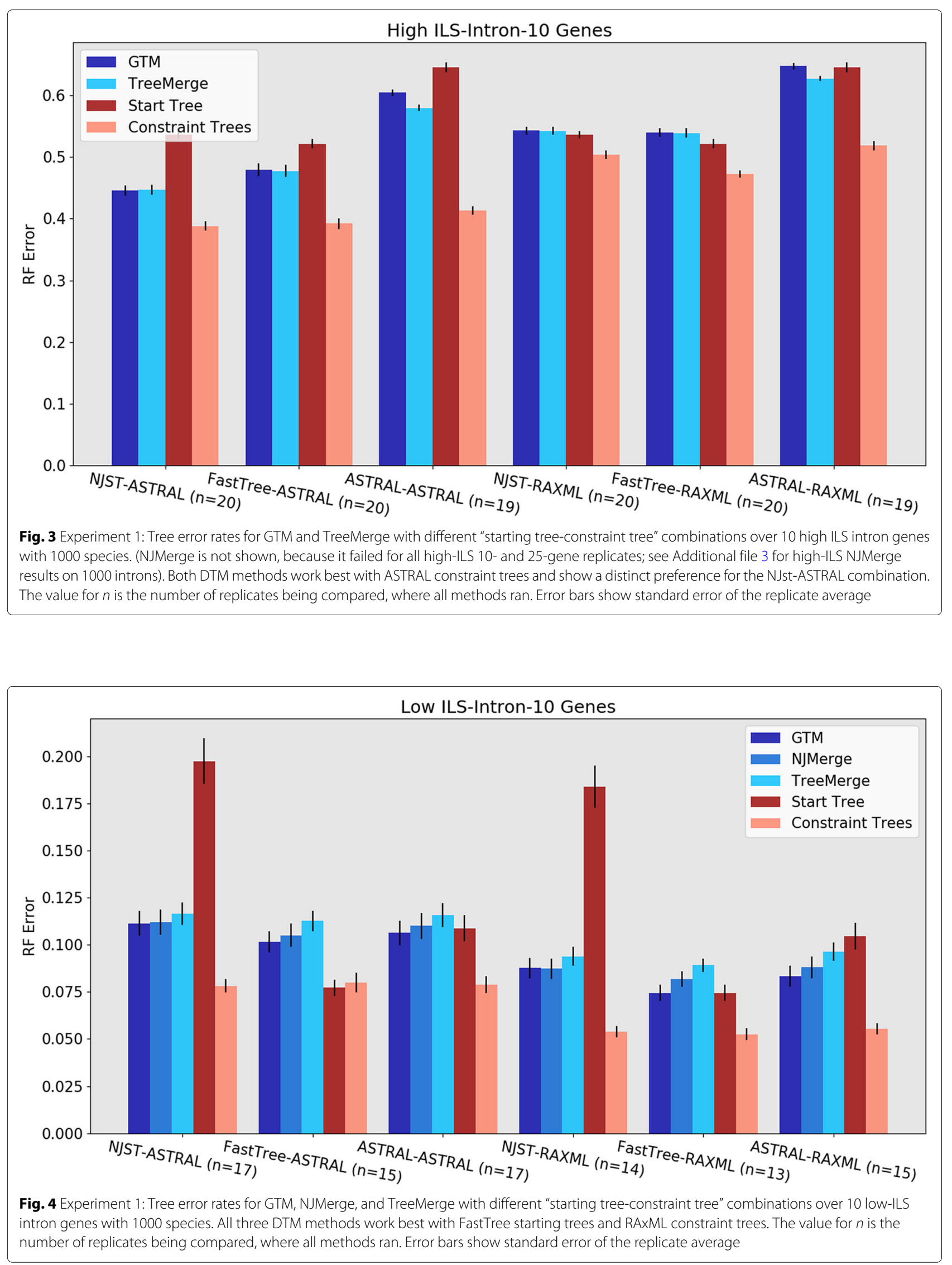


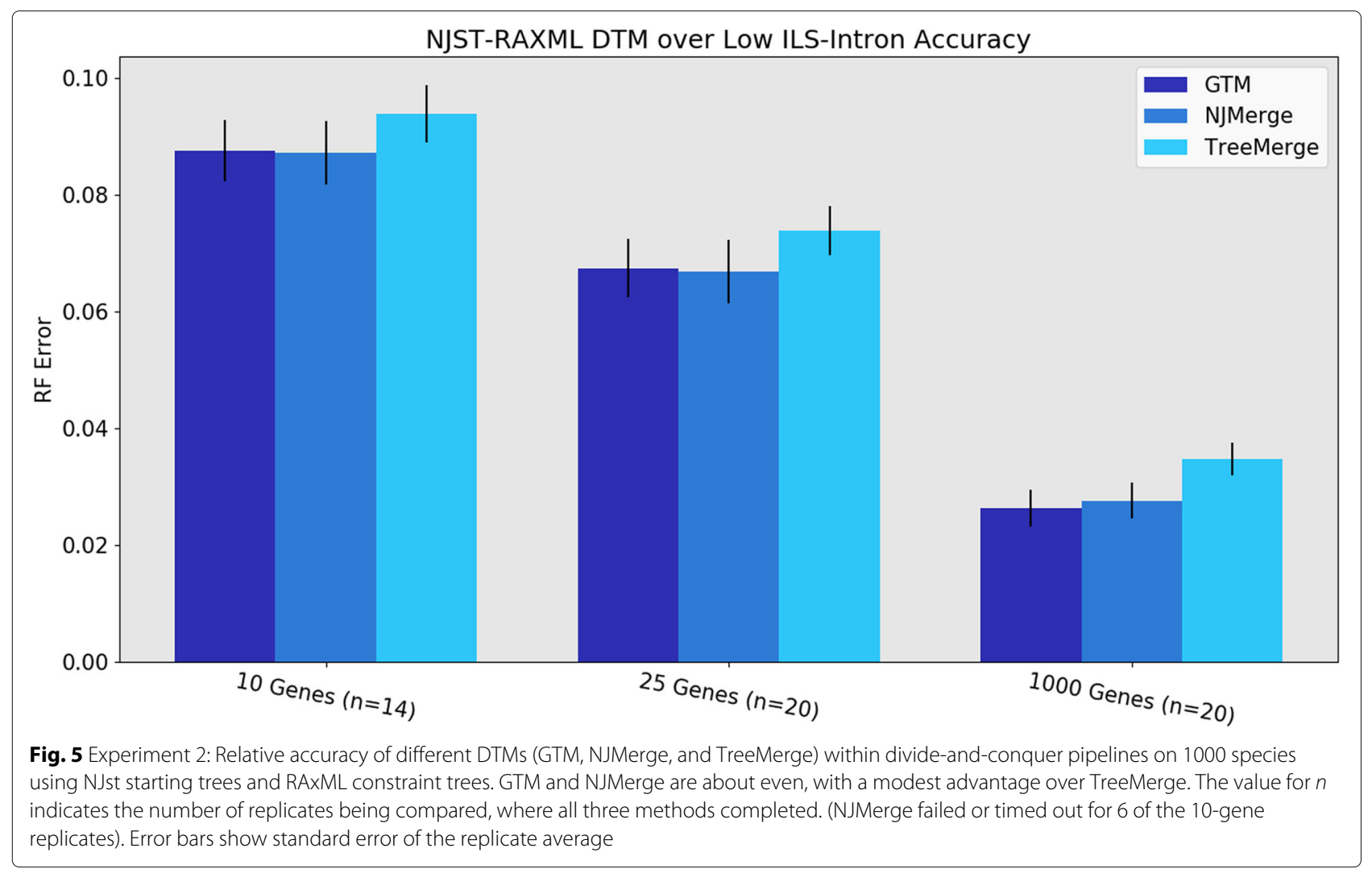

NJMerge, and up to 10 minutes for TreeMerge). However, GTM takes under a second for all model conditions, which represents an enormous speedup over the other two methods.

\section{Experiment 3: evaluating GTM-boosting}

One way of describing these GTM pipelines is that they are designed to improve (or "boost") the accuracy, speed, and/or scalability of a selected species tree estimation method used to compute constraint trees (which we refer to as the "base method"). Here, we explore the impact of "GTM-boosting" on ASTRAL and RAxML, two leading species tree estimation methods.

Table 2 Average runtime (seconds) on 1000-species datasets with high ILS, using NJst starting trees and ASTRAL constraint trees

\begin{tabular}{llll}
\hline & GTM & NJMerge & TreeMerge \\
\hline 10 Exons $(n=20)$ & 0.47 & $x$ & 655 \\
10 Introns $(n=20)$ & 0.55 & $x$ & 524 \\
25 Exons $(n=20)$ & 0.46 & $x$ & 504 \\
25 Introns $(n=20)$ & 0.46 & $x$ & 509 \\
1000 Exons $(n=20)$ & 0.47 & 1950 & N/A \\
1000 Introns $(n=20)$ & 0.47 & 1879 & N/A \\
\hline
\end{tabular}

The value for $n$ is the number of replicates being compared, where both GTM and TreeMerge finished. NJMerge and TreeMerge timings were unavailable for 1000 genes. NJMerge failed on all 10 and 25 -gene high ILS replicates

\section{Impact of GTM-boosting on ASTRAL}

ASTRAL is the leading method for species tree estimation when gene tree heterogeneity is the result of ILS, and it is statistically consistent under the MSC. Here we compare the NJst-ASTRAL-GTM pipeline (which is statistically consistent under the MSC) to ASTRAL, and also to ASTRID and NJst, two other summary methods that are also statistically consistent under the MSC.

Results for the intron datasets with high ILS (Fig. 6) show this pipeline has the best accuracy of all methods for 10 and 25 genes (with a large improvement especially for the 10-gene datasets), and then all four methods have essentially the same error rates on 1000 genes (with a

Table 3 Average runtimes (in seconds) on 1000-species datasets with low ILS, using NJst starting trees and RAxML constraint trees

\begin{tabular}{llll}
\hline & GTM & NJMerge & TreeMerge \\
\hline 10 Exons $(n=2)$ & 0.43 & 946 & 477 \\
10 Introns $(n=8)$ & 0.42 & 732 & 446 \\
25 Exons $(n=9)$ & 0.41 & 728 & 583 \\
25 Introns $(n=10)$ & 0.43 & 685 & 543 \\
1000 Exons $(n=20)$ & 0.43 & 2055 & N/A \\
1000 Introns $(n=20)$ & 0.43 & 2010 & N/A \\
\hline
\end{tabular}

The value for $n$ is the number of replicates being compared, where all three methods finished; NJMerge failed or timed out on the missing 10 and 25-gene low ILS replicates. TreeMerge timings were unavailable for 1000 genes 

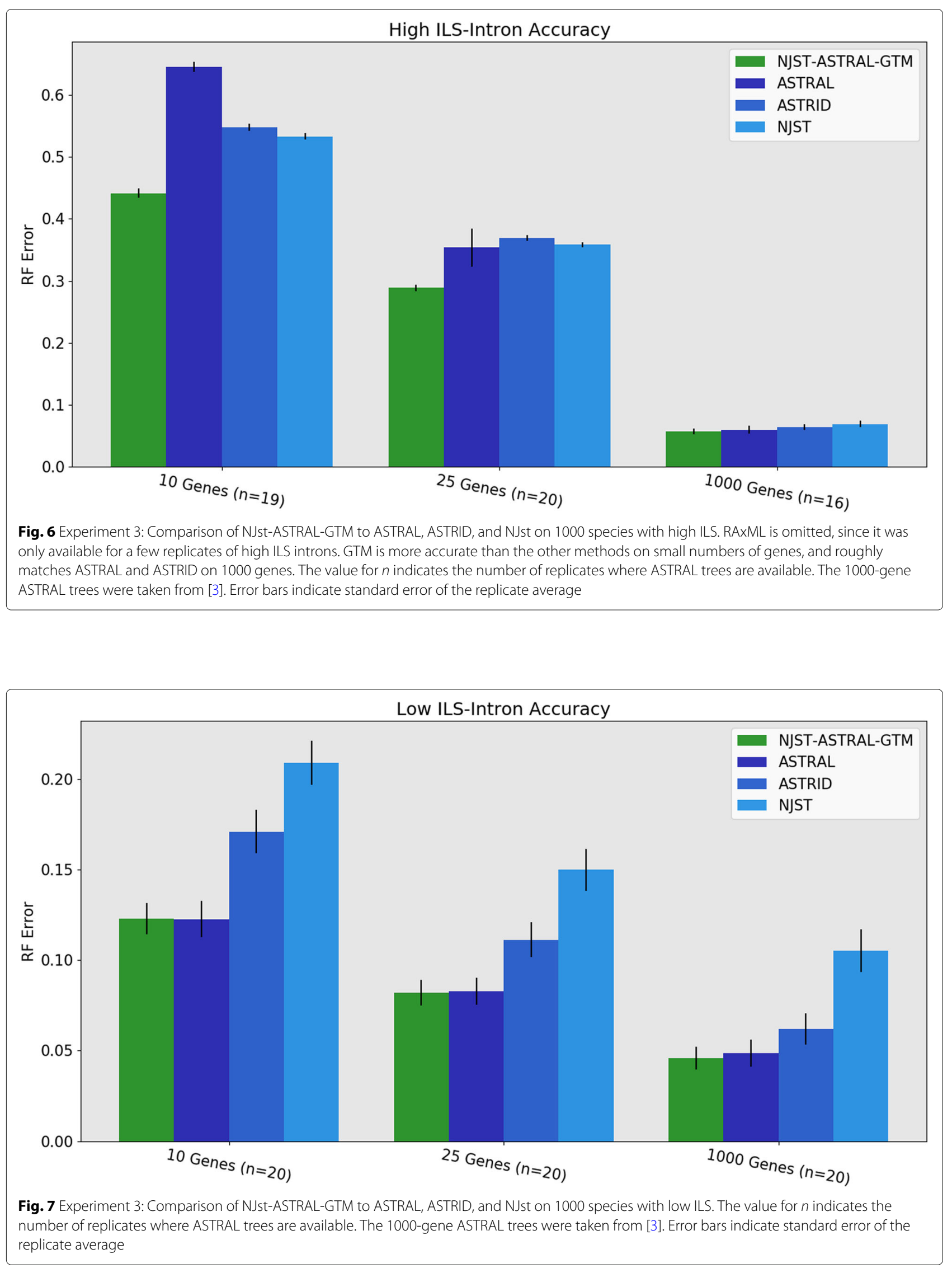
slight advantage to NJst-ASTRAL-GTM and ASTRAL). Analyses on the same datasets and including RAxML (shown in Additional file 3) reveal that RAxML is less accurate than the summary methods for these high ILS conditions.

Comparing the same set of summary methods under low ILS conditions (Fig. 7) shows ASTRAL and NJstASTRAL-GTM are essentially indistinguishable for accuracy and dominate the next best method, ASTRID, for all numbers of genes. The difference between NJst-ASTRALGTM and ASTRID is large for 10 genes and decreases for larger numbers of genes. Also, ASTRID clearly dominates NJst, with a large gap between the two methods for all numbers of genes.

Next, we compare the running time for NJst-ASTRALGTM and ASTRAL. Under high ILS conditions (see Table 4), ASTRAL took 2.4 hours on 10 introns whereas NJst-ASTRAL-GTM completed in 98 seconds, so that ASTRAL uses almost two orders of magnitude more time than NJst-ASTRAL-GTM. The magnitude of the reduction in running time decreased as the number of genes increased, but was still large for 1000 genes (42.5 hours, for ASTRAL and 2.2 hours for NJst-ASTRAL-GTM). These are dramatic savings in running time.

The difference is less dramatic under low ILS conditions, since ASTRAL runs much faster. Nevertheless, the pipeline runs a bit more than twice as fast on 10 genes, a bit less than twice as fast on 25 , and about twice as fast on 1000 (Table 5).

Table 4 Comparison of average runtime (seconds) of NJst-ASTRAL-GTM and ASTRAL for high ILS conditions with introns on 1000 species

\begin{tabular}{lll}
\hline & NJst-ASTRAL-GTM & ASTRAL \\
\hline 10 Genes $(\boldsymbol{n}=\mathbf{1 8})$ & & \\
-Pre-GTM & 97.4 & n.a. \\
-ASTRAL & n.a. & $8,617.0$ \\
-GTM & 0.4 & n.a. \\
-Total & 97.8 & $8,656.0$ \\
$\mathbf{2 5}$ Genes $(\boldsymbol{n = 2 0 )}$ & & \\
-Pre-GTM & 174.7 & n.a. \\
-ASTRAL & n.a. & $5,441.4$ \\
-GTM & 0.4 & n.a. \\
-Total & 175.1 & $5,539.4$ \\
$\mathbf{1 0 0 0}$ Genes $(\boldsymbol{n = 1 6 )}$ & & \\
-Pre-GTM & $7,948.9$ & n.a. \\
-ASTRAL & n.a. & $149,145.9$ \\
-GTM & 0.4 & n.a. \\
-Total & $7,949.3$ & $153,045.9$ \\
\hline
\end{tabular}

The value for $n$ is the number of replicates being compared (i.e., where ASTRAL trees are available). Pre-GTM covers computing gene trees using FastTree, the NJst starting tree, and ASTRAL subset trees; the gap between "total" and "ASTRAL" for the right hand column reflects the time to compute gene trees using FastTree, which is 3.9 seconds per gene. Results for the 1000-gene ASTRAL trees are taken from the NJMerge study [3]
Table 5 Comparison of average runtime (seconds) of NJst-ASTRAL-GTM and ASTRAL for low ILS conditions with introns on 1000 species

\begin{tabular}{lll}
\hline & NJst-ASTRAL-GTM & ASTRAL \\
\hline $\mathbf{1 0}$ Genes $(\boldsymbol{n = 2 0})$ & 59.1 & \\
-Pre-GTM & n.a. & n.a. \\
-ASTRAL & 0.4 & 114.4 \\
-GTM & 59.5 & n.a. \\
-Total & & 153.4 \\
$\mathbf{2 5}$ Genes $(\boldsymbol{n}=\mathbf{2 0})$ & 120.9 & \\
-Pre-GTM & n.a. & n.a. \\
-ASTRAL & 0.4 & 101.3 \\
-GTM & 121.3 & n.a. \\
-Total & & 199.3 \\
$\mathbf{1 0 0 0}$ Genes $(\boldsymbol{n}=\mathbf{1 9})$ & $4,308.4$ & \\
-Pre-GTM & n.a. & n.a. \\
-ASTRAL & 0.4 & 4,894.4 \\
-GTM & $4,308.8$ & n.a. \\
-Total & 8,794.4 \\
\hline
\end{tabular}

The value for $n$ is the number of replicates being compared (i.e., where ASTRAL trees are available). Pre-GTM covers computing gene trees using FastTree, the NJst starting tree, and ASTRAL subset trees; the gap between "total" and "ASTRAL" for the right hand column reflects the time to compute gene trees using FastTree, which is 3.9 seconds per gene. Results for the 1000-gene ASTRAL trees are taken from the NJMerge study [3]

\section{Impact of GTM-boosting on RAXML}

Concatenated analysis using maximum likelihood is one of the major approaches to species tree estimation. RAxML is considered by many to be the leading ML heuristic, and outperforms FastTree, a fast but less accurate maximum likelihood heuristic, with respect to maximum likelihood scores. However, FastTree can run on very large datasets, and can (in some conditions) match the topological accuracy of RAxML [27].

Here we address the potential for scaling RAxML to large datasets through the use of a divide-and-conquer pipeline using GTM. As we noted earlier, FastTreeRAxML-GTM has higher accuracy than NJst-RAxMLGTM but cannot complete (within the 4 hour time limit) on the 1000-gene datasets, and for this reason we also include NJst-RAxML-GTM. We compare these two versions of GTM-boosting to RAxML and FastTree, under both low and high ILS conditions.

When restricted to the datasets where FastTree completes, we see the following trends. Under low ILS, FastTree-RAxML-GTM provides substantially better accuracy than NJst-RAxML-GTM and is nearly identical to RAxML, although it is slightly worse than FastTree by itself (Fig. 8). Under high ILS, FastTree-RAxML-GTM and NJst-RAxML-GTM are indistinguishable for accuracy, and both are somewhat more accurate than RAxML 


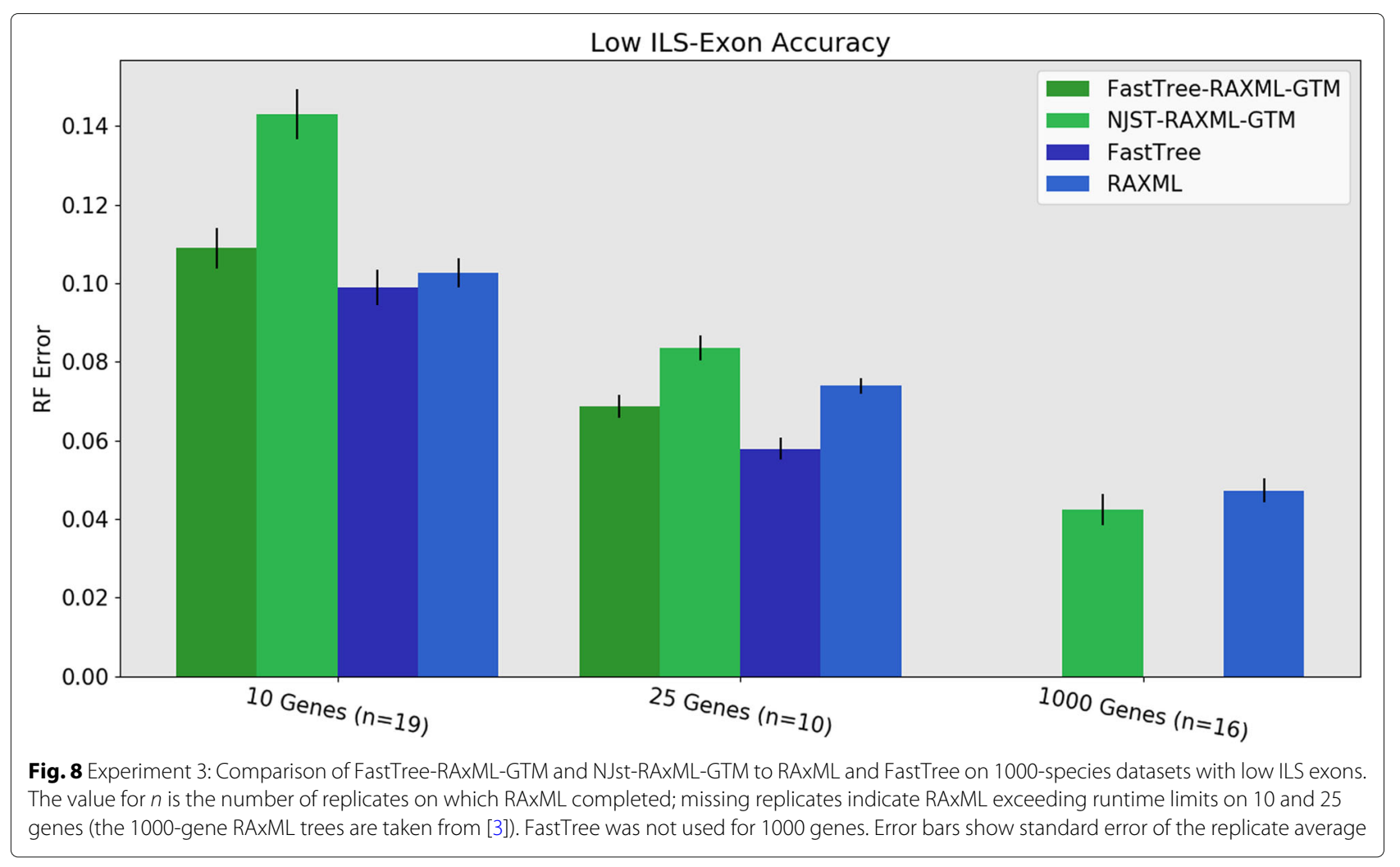

and less accurate than FastTree (Fig. 9). The very good accuracy of FastTree in comparison to RAxML is noteworthy, especially since RAxML is established to be a better maximum likelihood heuristic than FastTree. However, due to computational limitations in this study, we ran RAxML with only one random starting condition, instead of running it with a larger number (e.g., 10 or 20) of random starting conditions and then selecting the tree with the best ML score. This choice may have reduced the accuracy of RAxML relative to FastTree.

We now discuss the running time comparisons between RAxML and the two GTM pipelines that use RAxML to compute constraint trees. RAxML often failed to complete within the allowed time limit (4 hours) on many 10 - and 25-gene datasets. Results reported on the 1000gene datasets were obtained in [3], where RAxML was allowed to run for 48 hours, and the best found tree was returned. However, even on the 10-gene and 25gene datasets there are noteworthy differences, which we now discuss. FastTree-RAxML-GTM and NJst-RAxMLGTM completed on all the 10- and 25-gene datasets but RAxML completed on only 15/80 25-gene datasets and 55/80 10-gene datasets (Additional file 4).

When restricted to those datasets where RAxML completed within 4 hours, RAxML used (on average) between 2 and 3 hours, depending on the model condition (with more time needed for the high ILS datasets with 25 genes).
In comparison, the average running time on these datasets for the two GTM pipelines were much smaller: at most 27 minutes for NJst-RAxML-GTM and at most 36 minutes for FastTree-RAxML-GTM (e.g., see Table 6). Thus, NJst-RAxML-GTM is the fastest, followed (fairly closely) by FastTree-RAxML-GTM, and both are much faster than RAxML. However, because FastTree cannot run on the 1000-gene datasets, NJst-RAxML-GTM is the only scalable GTM pipeline that uses RAxML on subsets.

\section{Discussion}

The datasets generated for this study each have 1000 species but otherwise vary in terms of number of genes (10, 25, and 1000), ILS level (low and high), and type of gene (exon or intron). Thus, the model conditions provide a range of conditions that include many of the conditions observed in species tree estimation for large numbers of species. That said, the observations made in this study are limited to these conditions, and other conditions may show other trends.

The first overall observation we make is that GTM is comparable in accuracy to TreeMerge and NJMerge (but is much faster). We expected to see an improvement in running time over NJMerge, which is not designed to be very fast, but we did not expect to necessarily see an improvement in running time over TreeMerge, and the degree of improvement was larger than we might 


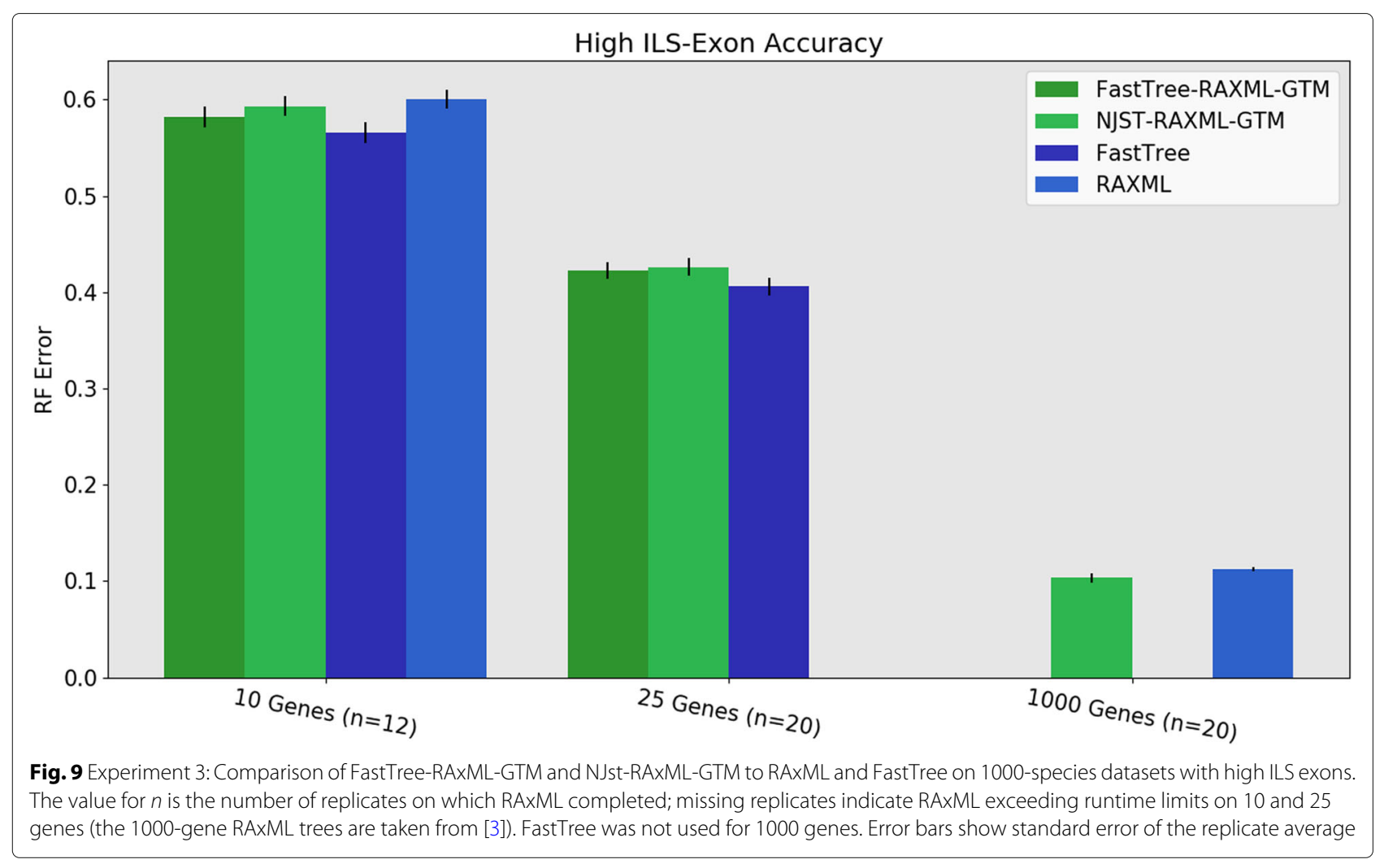

have expected. The observation that GTM is typically comparable in accuracy to TreeMerge and NJMerge is surprising because GTM cannot blend, while TreeMerge and NJMerge can.

We focus on the comparison between TreeMerge and GTM, since NJMerge failed on many datasets in our experiment. There are some conditions where GTM is slightly more accurate than TreeMerge and some other conditions where GTM is slightly less accurate than TreeMerge, and these conditions have different properties. Specifically, there can be a small advantage to GTM when the starting tree is not too poor (at most 60\% RF error) and a small advantage to TreeMerge when the starting tree has very poor accuracy (i.e., on the 10-gene high ILS datasets, where the ASTRAL starting trees had 6465\% RF error rates, see Fig. 3 and Additional file 3). Thus, the accuracy of the starting tree has an impact on the relative accuracy of GTM and TreeMerge. We expected to see GTM degrade in accuracy with poor starting trees (which is why we included conditions where poor starting trees were computed), and so this part is not surprising. However, what is surprising is that GTM matches the accuracy of TreeMerge even with fairly mediocre starting trees, including ones where the starting tree has $50-60 \%$ RF error. Overall, our study shows that the limitation of not being able to blend subset trees was not a significant problem for GTM, under the conditions we explored.
The next observations have to do with the impact of "GTM-boosting" on the method used to compute constraint trees (i.e., the "base method"). Our study shows that GTM-boosting provides substantial improvements in speed for all tested model conditions and generally maintained (and sometimes even improved) accuracy over the base method. The improvement in running time obtained by GTM-boosting was expected, as previous divide-andconquer approaches have shown similar trends [28, 29].

The improvement in accuracy seen in NJst-ASTRALGTM over ASTRAL and NJst-RAxML-GTM or FastTreeRAxML-GTM over RAxML are surprising, and worth trying to understand.

The conditions in which NJst-RAxML-GTM or FastTree-RAxML-GTM were more accurate than RAxML were cases with 25 genes, where they produced very slightly better results; for other numbers of genes, RAxML was more accurate. Somewhat similar trends were observed in [3] when NJMerge was used with RAxML in the same pipeline as we used here and found to produce somewhat more accurate trees than RAxML. The explanation offered in [3] was that NJmerge+RAxML is a combination of a coalescent-based species tree estimation method (the starting tree) and concatenation analysis method (the constraint trees), and the combination allows it to be more accurate than a method that is purely concatenation-based. In our study, this advantage 
Table 6 Average runtime (seconds) of FastTree-RAxML-GTM (GTM(RAxML)) and RAxML on 1000-species exon datasets

\begin{tabular}{|c|c|c|}
\hline & GTM(RAxML) & RAXML \\
\hline \multicolumn{3}{|c|}{ Low ILS 10 Genes ( $n=19)$} \\
\hline -FastTree & 279.6 & n.a. \\
\hline -RAxML subtrees & 831.3 & n.a. \\
\hline -GTM & 0.4 & n.a. \\
\hline -Total & $1,111.3$ & $7,313.7$ \\
\hline \multicolumn{3}{|c|}{ Low ILS 25 Genes $(n=10)$} \\
\hline -FastTree & 686.3 & n.a. \\
\hline -RAxML subtrees & $1,460.6$ & n.a. \\
\hline -GTM & 0.4 & n.a. \\
\hline -Total & $2,147.3$ & $10,539.4$ \\
\hline \multicolumn{3}{|c|}{ High ILS 10 Genes ( $n=12$ ) } \\
\hline -FastTree & 283.7 & n.a. \\
\hline -RAxML subtrees & 637.5 & n.a. \\
\hline -GTM & 0.4 & n.a. \\
\hline -Total & 921.6 & $10,135.6$ \\
\hline \multicolumn{3}{|c|}{ High ILS 25 Genes ( $n=20$ ) } \\
\hline -FastTree & 731.5 & n.a. \\
\hline -RAxML subtrees & 1363.1 & n.a. \\
\hline -GTM & 0.4 & n.a. \\
\hline -Total & 2,095 & n.a. \\
\hline
\end{tabular}

The value for $n$ is the number of replicates being compared, i.e., where a RAxML tree is available

does not hold for the smaller numbers of genes, where NJst-RAxML-GTM is much less accurate than RAxML for 10 genes under the low ILS condition. Our explanation for why NJst-RAxML-GTM is substantially less accurate than RAxML for 10-gene low ILS datasets is that the NJst starting tree has high error (Additional file 3). This is consistent with the observations that FastTreeRAxML-GTM is close to RAxML for accuracy on low ILS conditions, even with 10 genes, and the FastTree starting tree also has high accuracy. Thus, as we have seen, the starting tree has an impact on accuracy, and the choice of starting tree should reflect the level of ILS.

The conditions in which NJst-ASTRAL-GTM was more accurate than ASTRAL occur for the high ILS model conditions with ten genes. Frankly, this improvement in accuracy is surprising and was not expected. However, Fig. 3 and Additional file 3) provide some insight. The difference in tree error for ASTRAL on the full dataset (i.e., as the starting tree) and on the subsets (i.e., as the constraint tree method) are dramatic for both introns and exons. For example, ASTRAL had average 64\% RF error on 10 introns but the average ASTRAL constraint tree error was only $39 \%$ error-a large drop in error rate. In other words, ASTRAL was more accurate on the smaller subsets (each with at most 120 species) than it was with the full dataset (with 1000 species). One possible explanation for this trend is that when the number of genes is very small, the constrained search strategy employed by ASTRAL works better with a smaller number of species.

GTM using appropriate constraint tree methods (i.e., ASTRAL for high ILS and RAxML for low ILS) nearly always improved on the starting tree, often substantially. The only exceptions to this rule are the low ILS conditions with 10 or 25 genes where the best starting trees had very low error (Fig. 4, and also figures in Additional file 3). Even for these cases, using GTM matched the starting tree accuracy except for one model condition (25 low ILS exon datasets) where it produced a tree with $1 \%$ higher error (Additional file 3). In contrast, the other DTMs reduced accuracy compared to these highly accurate starting trees.

Our study also showed that GTM divide-and-conquer pipelines reduce the running time and enable expensive base methods (here, RAxML and ASTRAL) to be applied to large datasets. The improvement in running time is most noticeable for the high ILS conditions, where ASTRAL and RAxML require more time. Furthermore, for all numbers of genes and ILS conditions, the vast majority of the time used within a GTM pipeline is the pre-GTM component (which computes the gene trees, the starting tree, and the subset trees); in contrast, the GTM part used approximately half a second, even on 1000 genes and 1000 species.

A basic question that is relevant to GTM divide-andconquer pipelines is the sensitivity of the approach to the algorithmic parameters, including the starting tree (which is also used as the guide tree), the method used to compute constraint trees, and even the decomposition strategy. Our study shows that the starting tree and the constraint tree method both have an impact on the accuracy of the final tree. For example, we noted that FastTree-RAxMLGTM is more accurate than NJst-RAxML-GTM on low ILS datasets (reflecting the improved accuracy of FastTree for low ILS conditions), and that NJst-ASTRAL-GTM is more accurate than NJst-RAxML-GTM on high ILS datasets (reflecting the improved accuracy of ASTRAL as a constraint tree method for high ILS conditions). However, we did not evaluate changes to the decomposition strategy (e.g., changing the subset size or which edges we delete) nor did we examine the potential impact of allowing the guide tree to be different from the starting tree.

One obvious case where the starting tree and guide tree could be different is where GTM is used within an iterative strategy, where each iteration uses the tree computed during the previous iteration for the starting tree, divides into subsets, constructs trees on the subsets, and then merges the constraint trees using a fixed guide tree. In such a scenario, the guide tree is fixed but the "starting tree" (which 
is only used to compute the decomposition) can change in each iteration. In this context the guide tree only impacts the merger step and not also the decomposition strategy, and the impact of the guide tree (when it is used with a good starting tree) is an interesting question. For example, if the decomposition is based on the true tree and the constraint trees are the true tree on the subsets, then how much can a bad guide tree impact the outcome? A simple thought experiment, based on only dividing into two subsets, reveals that the impact may not be minor. For example, consider the case where the true tree is a caterpillar tree with $n$ leaves (i.e., a path of length $n-3$ with the leaves hanging off the path) and the division is into two subsets. Given a random guide tree, GTM would combine the two trees by subdividing a random edge in each of the two constraint trees (thus creating two nodes, $v_{1}$ and $v_{2}$ ), and attaching the two trees by adding a new edge between $v_{1}$ and $v_{2}$. Note that such a randomly merged tree would have high error, even though the constraint trees and the starting tree are completely accurate. Thus, a random guide tree can result in high error, though the degree of error will depend on the topology of the true tree, the decomposition strategy (and in particular the number of subsets), and the error in the constraint trees.

\section{Summary and conclusions}

Divide-and-conquer phylogeny estimation using Disjoint Tree Merger (DTM) is a recently developed approach to large-scale tree estimation that has been shown to improve speed while maintaining (and in some cases improving) accuracy for large-scale phylogenomic datasets, and maintaining statistical consistency under the MSC+GTR model. Here, we proposed the DTM-GT problem, which seeks a compatibility tree that is as close as possible to an input "guide tree". We proved that DTMGT is NP-hard, and presented GTM, which solves the problem exactly and in polynomial time if blending is not allowed. Our experimental study showed that GTM generally matches or improves on the accuracy of two prior DTM methods, TreeMerge and NJMerge, while being much faster. Therefore, although GTM has the vulnerability of being unable to blend (a limitation that neither TreeMerge nor NJMerge have), this vulnerability had limited impact in this study.

Importantly, GTM-boosting improved the accuracy and speed of ASTRAL, a leading method for species tree estimation that addresses gene tree heterogeneity due to incomplete lineage sorting. GTM-boosting also improved the speed for RAxML, the leading heuristic for concatenated maximum likelihood, while coming very close to it in accuracy (and sometimes being even more accurate) when used with FastTree as the starting tree. Thus, GTMboosting provides substantial advances for multi-locus species tree estimation on large numbers of species.
Our study reveals the surprising potential of unblended DTM methods, and suggests many directions for future research. For example, GTM is just the first unblended DTM, and it seems likely that other unblended DTMs might be more accurate. Since GTM is impacted by its guide tree, unblended DTMs that are not based on guide trees might be more accurate.

GTM itself could be modified to allow for blended merging, with potential improvement in accuracy. For example, the refinement step in GTM where the collapsed tree is refined to separate the constraint sets is very simple, and is performed to ensure an unblended merger. However, if blending were permitted, then other ways of refining the tree could be considered (provided that they do not produce trees that violate the constraint trees). Polytomy refinement is a natural problem in phylogenetics (e.g., [30]), and has also been specifically discussed in the context of gene tree correction given a species tree; see, for example, [31] (and references therein) for a discussion of refinement techniques that address ILS and [32] (and references therein) for polytomy refinement techniques that address gene duplication and loss.

DTM methods (blended or unblended) can also be used for other phylogeny estimation problems where the most accurate base methods are computationally intensive. For example, DTMs could also be used with Bayesian methods to co-estimate gene trees and species trees [33] or for "genome rearrangement phylogeny", which takes chromosomal rearrangements, duplications, and other events that change the chromosomal architecture into account.

Furthermore, a parallel implementation of the divideand-conquer pipeline could have high impact. As our study showed, the most expensive part of these divideand-conquer pipelines is the computation of the constraint trees. With sufficient parallelism, these analyses could become very fast, and each iteration could complete quickly, leading potentially to the ability to compute species trees on ultra-large datasets (with thousands of genes and species) within a few hours.

Finally, we note that there are cases where an adequately accurate starting tree cannot be estimated using currently available fast methods. Examples of such situations include the challenge of estimating a tree from ultra-large datasets of unaligned sequences where alignment estimation is difficult due to sequence heterogeneity and size; this challenge is exacerbated when the datasets include fragmentary sequences, which are not only difficult to align but can reduce accuracy for tree estimation $[34,35]$. For such data, standard two-phase methods that first compute an alignment and then compute a tree do not have acceptable accuracy, while PASTA [16], BAli-Phy [36], and other co-estimation methods are not fast. It is possible that alignment-free methods (see [37-39] for an entry into this topic) might provide 
good starting trees, but these have not been tested on ultra-large datasets (with thousands of species), and have instead mainly been focused on genome-scale analyses of tens of genomes. However, for any large dataset on which the starting trees cannot be reasonably accurately estimated quickly, blended DTM divide-and-conquer strategies may provide the best accuracy. Thus, future work into developing new DTMs, both blended or unblended, is merited.

We close with some comments about the implications of this study for large-scale phylogenomic analysis. As shown in this study, the choice of method for species tree estimation depends on the level of ILS and even the number of genes. The results for low ILS datasets suggest that maximum likelihood heuristics, such as FastTree and RAxML, or using GTM-boosting with fast ML heuristics for the starting tree and more accurate ML heuristics for constraint trees, may be advantageous. Of interest is the relative performance of RAxML and FastTree (which favored FastTree in our experiments), but we note that RAxML was not run so as to obtain the best accuracy, and so further study is needed. Results for high ILS datasets are more definitive: GTMboosting, using NJst as the starting tree and ASTRAL for constraint trees, matched or improved on ASTRAL for all model conditions and numbers of genes, and was always faster. Thus, for high ILS conditions, GTMboosting provides a distinct advantage for both accuracy and time.

\section{Supplementary information}

Supplementary information accompanies this paper at https://doi.org/10.1186/s12864-020-6605-1.

Additional file 1: Codes used in the performance study. This document provides the commands used to perform the simulation study.

Additional file 2: Additional proofs. This document provides proofs of Theorems 1-3.

Additional file 3: Additional figures. This document provides additional figures for the performance study.

Additional file 4: Additional tables. This document provides additional tables for the performance study.

\section{Acknowledgements}

The authors thank Sarah Christensen, Erin Molloy, Pranjal Vachaspati, and Xilin Yu for helpful comments.

\section{About this supplement}

This article has been published as part of BMC Genomics Volume 21 Supplement 2, 2020: Proceedings of the 17th Annual Research in Computational Molecular Biology (RECOMB) Comparative Genomics Satellite Workshop: genomics. The full contents of the supplement are available online at https://bmcgenomics. biomedcentral.com/articles/supplements/volume-21-supplement-2

\section{Authors' contributions}

TW directed the research. VL designed and implemented the algorithm, performed the experimental study, and created the figures. VL and TW proved the theorems and wrote the paper. All authors read and approved the final manuscript.

\section{Funding}

This research was supported by NSF grants 1513629 and 1535977 to TW. Publication costs are funded by NSF grant 1535977.

\section{Availability of data and materials}

Datasets used can be found at https://databank.illinois.edu/datasets/IDB3204101 and https://databank.illinois.edu/datasets/IDB-1424746. GTM software available at https://github.com/vlasmirnov/GTM.

Ethics approval and consent to participate

Not applicable.

\section{Consent for publication}

Not applicable.

\section{Competing interests}

The authors declare that they have no competing interests.

Published: 16 April 2020

\section{References}

1. Warnow T. Divide-and-conquer tree estimation: Opportunities and challenges. In: Warnow T, editor. Bioinformatics and Phylogenetics: Seminal Contributions of Bernard Moret. Cham: Springer; 2019. p. 121-50.

2. Molloy EK, Warnow T. NJMerge: A Generic Technique for Scaling Phylogeny Estimation Methods and Its Application to Species Trees. In: Blanchette M, Ouangraoua A, editors. Comparative Genomics. RECOMB-CG 2018. Lecture Notes in Computer Science vol. 11183. Cham: Springer; 2018. https://doi.org/10.1007/978-3-030-00834-5_15.

3. Molloy EK, Warnow T. Statistically consistent divide-and-conquer pipelines for phylogeny estimation using NJMerge. Algoritm Mol Biol. 2019;14(1):14. https://doi.org/10.1186/s13015-019-0151-x.

4. Molloy EK, Warnow T. TreeMerge: A new method for improving the scalability of species tree estimation methods. Bioinformatics. 2019. Special issue for ISMB 2019, https://doi.org/10.1093/bioinformatics/ btz344.

5. Zhang Q, Rao S, Warnow T. Constrained incremental tree building: new absolute fast converging phylogeny estimation methods with improved scalability and accuracy. Algoritm Mol Biol. 2019;14(1):2.

6. Le T, Sy A, Molloy EK, Zhang QR, Rao S, Warnow T. Using inc within divide-and-conquer phylogeny estimation. In: International Conference on Algorithms for Computational Biology. Springer; 2019. p. 167-78. https://doi.org/10.1007/978-3-030-18174-1_12.

7. Mirarab S, Reaz R, Bayzid MS, Zimmermann T, Swenson MS, Warnow T. ASTRAL: genome-scale coalescent-based species tree estimation. Bioinformatics. 2014;30(17):541-8. https://doi.org/10.1093/ bioinformatics/btu462.

8. Mirarab S, Warnow T. ASTRAL-II: coalescent-based species tree estimation with many hundreds of taxa and thousands of genes. Bioinformatics. 2015;31(12):44-52. https://doi.org/10.1093/bioinformatics/btv234.

9. Zhang C, Rabiee M, Sayyari E, Mirarab S. ASTRAL-III: polynomial time species tree reconstruction from partially resolved gene trees. BMC Bioinformatics. 2018;19(6):153. https://doi.org/10.1186/s12859-018-2129y.

10. Stamatakis A. RAxML-VI-HPC: maximum likelihood-based phylogenetic analyses with thousands of taxa and mixed models,. Bioinformatics. 2006;22(21):2688-90.

11. Maddison WP. Gene trees in species trees. Syst Biol. 1997;46(3):523-36. https://doi.org/10.1093/sysbio/46.3.523.

12. Saitou N, Nei M. The neighbor-joining method: a new method for reconstructing phylogenetic trees. Mol Biol Evol. 1987;4(4):406-25. https://doi.org/10.1093/oxfordjournals.molbev.a040454.

13. Liu L, Yu L. Estimating Species Trees from Unrooted Gene Trees. Syst Biol. 2011;60(5):661-7. https://doi.org/10.1093/sysbio/syr027.

14. Liu K, Warnow TJ, Holder MT, Nelesen SM, Yu J, Stamatakis AP, Linder CR. SATe-ll: very fast and accurate simultaneous estimation of multiple sequence alignments and phylogenetic trees. Syst Biol. 2011;61(1):90.

15. Mirarab S, Nguyen N, Warnow T. PASTA: ultra-large multiple sequence alignment. In: International Conference on Research in Computational 
Molecular Biology (RECOMB). Springer; 2014. p. 177-91. https://doi.org/ 10.1007/978-3-319-05269-4_15.

16. Mirarab S, Nguyen N, Guo S, Wang L-S, Kim J, Warnow T. PASTA: ultra-large multiple sequence alignment for nucleotide and amino-acid sequences. J Comput Biol. 2015;22(5):377-86.

17. Nelesen S, Liu K, Wang L-S, Linder CR, Warnow T. DACTAL: divide-and-conquer trees (almost) without alignments. Bioinf. 2012;28(12):274-82. https://doi.org/10.1093/bioinformatics/bts218.

18. Maddison WP. Gene Trees in Species Trees. Syst Biol. 1997;46(3):523-36. https://doi.org/10.1093/sysbio/46.3.523.

19. Tavaré S. Some probabilistic and statistical problems in the analysis of DNA sequences. In: Lectures on Mathematics in the Life Sciences vol 17. Providence, RI: American Mathematical Society; 1986. p. 57-86.

20. Price MN, Dehal PS, Arkin AP. FastTree 2 - Approximately Maximum-Likelihood Trees for Large Alignments. PLOS ONE. 2010;5(3): 1-10. https://doi.org/10.1371/journal.pone.0009490.

21. Roch S, Steel MA. Likelihood-based tree reconstruction on a concatenation of aligned sequence data sets can be statistically inconsistent. Theor Popul Biol. 2015;100:56-62.

22. Roch S, Nute M, Warnow T. Long-Branch Attraction in Species Tree Estimation: Inconsistency of Partitioned Likelihood and Topology-Based Summary Methods. Syst Biol. 2018;68(2):281-97. https://doi.org/10.1093/ sysbio/syy061.

23. Robinson D, Foulds L. Comparison of phylogenetic trees. Math Biosci. 1981;53(1-2):131-47.

24. Vachaspati P, Warnow T. ASTRID: Accurate Species TRees from Internode Distances. BMC Genomics. 2015;16(10):3. https://doi.org/10.1186/14712164-16-S10-S3.

25. Warnow T, et al. Illinois Data Bank repository for the Warnow Laboratory at the University of Illinois. 2019. https://databank.illinois.edu/datasets? sort_by=sort_updated_desc\&q=Warnow\&per_page $=25$. Last Accessed 15 Aug 2019

26. Price MN, Dehal PS, Arkin AP. FastTree 2-approximately maximum-likelihood trees for large alignments. PloS one. 2010;5(3):9490.

27. Liu K, Linder CR, Warnow T. RAxML and FastTree: comparing two methods for large-scale maximum likelihood phylogeny estimation. PLoS ONE. 2012;6(11):27731.

28. Bayzid MS, Hunt T, Warnow T. Disk-Covering Methods Improve Phylogenomic Analyses. BMC Genomics. 2014;15(Suppl 6):7. Proceedings of RECOMB-CG (Comparative Genomics).

29. Nelesen S, Liu K, Wang L-S, Linder CR, Warnow T. DACTAL: divide-and-conquer trees (almost) without alignments. Bioinformatics. 2012;28(12):274-82

30. Bonet M, Steel M, Warnow T, Yooseph S. Better methods for solving parsimony and compatibility. J Comput Biol. 1998;5(3):391-407.

31. Nakhleh L. Computational approaches to species phylogeny inference and gene tree reconciliation. Trends Ecol Evol. 2013;28(12):719-28.

32. Lafond M, Chauve C, Dondi R, El-Mabrouk N. Polytomy refinement for the correction of dubious duplications in gene trees. Bioinformatics. 2014;30(17):519-26.

33. Boussau B, Szöllősi GJ, Duret L, Gouy M, Tannier E, Daubin V. Genome-scale coestimation of species and gene trees. Genome Res. 2013;23(2):323-30.

34. Nguyen N, Mirarab S, Kumar K, Warnow T. Ultra-large alignments using phylogeny-aware profiles. Genome Biol. 2015;16(1):124.

35. Sayyari E, Whitfield JB, Mirarab S. Fragmentary gene sequences negatively impact gene tree and species tree reconstruction. Mol Biol Evol. 2017;34(12):3279-91.

36. Suchard MA, Redelings BD. BAli-Phy: simultaneous Bayesian inference of alignment and phylogeny. Bioinformatics. 2006;22(16):2047-8.

37. Criscuolo A. A fast alignment-free bioinformatics procedure to infer accurate distance-based phylogenetic trees from genome assemblies. Res Ideas Outcomes. 2019;5:36178.

38. Thankachan SV, Chockalingam SP, Liu Y, Krishnan A, Aluru S. A greedy alignment-free distance estimator for phylogenetic inference. BMC Bioinformatics. 2017;18(8):238. https://doi.org/10.1186/s12859-0171658-0.

39. Zielezinski A, Girgis HZ, Bernard G, Leimeister C-A, Tang K, Dencker T, Lau AK, Röhling S, Choi J, Waterman MS, et al. Benchmarking of alignment-free sequence comparison methods. BioRxiv. 2019611137. https://doi.org/10.1101/611137.

\section{Publisher's Note}

Springer Nature remains neutral with regard to jurisdictional claims in published maps and institutional affiliations.
Ready to submit your research? Choose BMC and benefit from:

- fast, convenient online submission

- thorough peer review by experienced researchers in your field

- rapid publication on acceptance

- support for research data, including large and complex data types

- gold Open Access which fosters wider collaboration and increased citations

- maximum visibility for your research: over $100 \mathrm{M}$ website views per year

At BMC, research is always in progress.

Learn more biomedcentral.com/submissions 